\title{
ACTINOBACILLUS PLEUROPNEUMONIAE SEROTYPES IN HUNGARY
}

\author{
Rita SÁRKÖZI, László MAKRAI and László FODOR ${ }^{*}$ \\ Department of Microbiology and Infectious Diseases, University of Veterinary Medicine, \\ H-1581 Budapest, P.O.B. 22, Hungary
}

(Received 20 December 2017; accepted 25 July 2018)

\begin{abstract}
A total of 255 Actinobacillus pleuropneumoniae isolates were collected from 634 lung samples representing 70 swine herds in Hungary between January 2012 and June 2016. On the basis of the indirect haemagglutination test 77 independent strains were included in the evaluation after the elimination of duplicate or multiple serotypes from the same herd. In the case of 7 herds strains of two different serotypes were identified. Fourteen Hungarian A. pleuropneumoniae isolates from the culture collection of the Department of Microbiology and Infectious Diseases, isolated before 2012, were also included in the evaluation (one each from 12 herds and two each from two herds, where two serotypes occurred). Out of the altogether $91 \mathrm{~A}$. pleuropneumoniae strains 72 strains belonged to biotype I and 19 strains could be allocated to biotype II. In Hungary, the most common serotypes were serotype $2(39.5 \%), 13(15.4 \%), 8(8.8 \%)$ and $16(8.8 \%)$, but serotypes $9(5.5 \%), 11(3.3 \%)$ and $12(3.3 \%)$ were also isolated. Twelve strains $(13.2 \%)$ were untypable.
\end{abstract} Hungary

Key words: Actinobacillus pleuropneumoniae, distribution of serotypes,

The porcine respiratory disease complex causes serious problems and huge economic losses in the swine industry throughout the world. One of the most important respiratory agents is Actinobacillus pleuropneumoniae. It is responsible for haemorrhagic-necrotic pneumonia and fibrinous pleuritis in growing and fattening pigs (Chiers et al., 2010; Ózsvári and Búza, 2015). In the presence of predisposing factors such as nutritional deficiencies, management problems and sometimes bacterial and viral infections, A. pleuropneumoniae can cause severe acute and chronic respiratory disease. It has two biotypes: biotype I strains need

*Corresponding author; E-mail: fodor.laszlo@univet.hu; Phone: 0036 (1) 251-9900; Fax: 0036 (1) 251-9260

Open Access. This is an open-access article distributed under the terms of the Creative Commons AttributionNonCommercial 4.0 International License (https://creativecommons.org/licenses/by-nc/4.0/), which permits unrestricted use, distribution, and reproduction in any medium for non-commercial purposes, provided the original author and source are credited, a link to the CC License is provided, and changes - if any - are indicated. 
nicotinamide adenine dinucleotide (NAD, V factor) for growth, while biotype II strains grow without NAD. The agent has important virulence factors: among others Apx toxins (RTX toxins), fimbriae, outer membrane proteins, ability of biofilm formation, presence of transporter systems and different enzymes are involved in the pathogenesis of pleuropneumonia (Chiers et al., 2010; Grasteau et al., 2011).

On the basis of surface soluble capsular polysaccharide antigens 18 serovars have been described so far (Nielsen, 1986a,b; Fodor et al., 1989; Nielsen et al., 1997; Blackall et al., 2002; Sárközi et al., 2015; Bossé et al., 2018). Several classical methods have been used for serotyping A. pleuropneumoniae strains, including slide agglutination, latex agglutination, complement fixation, indirect haemagglutination (IHA), coagglutination, ring and agar gel precipitation tests (Mittal et al., 1983a,b, 1987; Molnár, 1990; Nielsen et al., 1997). The IHA test is regarded to be the most specific and sensitive one among the classical serotyping methods (Mittal et al., 1983a and 1983b; Molnár, 1990; Nielsen et al., 1997). Cross reactions between serovars 1, 9 and 11, serovars 3, 6 and 8, and serovars 4 and 7 have been reported, which make serotyping rather difficult (Mittal et al., 1988; Zhou et al., 2008). Most conventional herds are infected with one or more serotypes of A. pleuropneumoniae, but in several cases only strains of low virulence are present (Gottschalk, 2012).

The frequency of the different serotypes and biotypes of A. pleuropneumoniae shows great geographical differences. Serotype 2 strains of A. pleuropneumoniae, which are dominant in Europe, produce two different exotoxins, while serotype 2 strains isolated in North America and Canada produce only one toxin (Gottschalk, 2012). Serotypes 2, 4 and 7 are the most common in Spain (Maldonado et al., 2009) and serotype 2 is the most frequent in Switzerland (Stärk et al., 2007). Serotypes 2, 3, 6-8, 10, 12 and 9 was reported to be dominant in the United Kingdom (Bossé et al., 2014), while serovar 15 was the leading type in Australia (Blackall et al., 2002). Serotypes 5, 7, 8 were the most frequently isolated types in Canada (Gottschalk and Lacouture, 2015). Biotype II strains are more commonly isolated in Europe, e. g. in Hungary (Fodor et al., 1989), in Denmark (Nielsen et al., 1997) and in Spain (Maldonado et al., 2009) than in the US (Perry et al., 2011).

Molecular biological methods are used more and more frequently to replace classical serotyping tests. Correlation between serovars, virulence and toxin production of the A. pleuropneumoniae strains described several years ago (Kamp et al., 1994; Frey et al., 1995; Marsteller and Fenwick, 1999) contributes to serotyping by detecting toxin genes; however, strains with atypical toxin production can give misleading results (Rayamajhi et al., 2005; Gottschalk, 2015). Identification of genes involved in the biosynthesis of capsular polysaccharides (Angen et al., 2008; Bossé et al., 2014; Marois-Crehan et al., 2014) and amplified fragment length polymorphism (AFLP) analysis (Kokotovic and Angen, 2007) can also be used for serotyping. 
Great changes have occurred in the agricultural sector in Hungary. Earlier serotype 2 was found to be the dominant serotype in Hungary, and serotypes 1, $5,8,10,11$ and 12 were also detected using the coagglutination test (Molnár, 1990). However, in recent decades the international trade in the Hungarian pig industry has intensified and there is no updated publication on the frequency of A. pleuropneumoniae serotypes. Therefore, the aim of this study was to examine the recent distribution and frequency of the different serotypes of A. pleuropneumoniae in Hungary using the indirect haemagglutination test.

\section{Materials and methods}

\section{Bacteria}

A total of 255 A. pleuropneumoniae isolates were collected from 634 lung samples representing 70 swine herds in Hungary between January 2012 and June 2016. On the basis of the indirect haemagglutination test 77 independent strains were included in the evaluation after the elimination of duplicate or multiple serotypes from the same herd. In the case of 7 herds strains of two different serotypes were identified. Furthermore, 14 Hungarian A. pleuropneumoniae isolates from the culture collection of the Department of Microbiology and Infectious Diseases, isolated before 2012, were also included in the evaluation (one each from 12 herds and two each from two herds, where two serotypes occurred), making the number of total isolates 91 as reported in the following sections.

Reference strains of serovars 1-15 of A. pleuropneumoniae were kindly provided by Dr. $\varnothing$. Angen (Danish Veterinary Laboratory, Copenhagen), serotype 16 is an own isolate (A-85/14) (Sárközi et al., 2015), while serotypes 17 and 18 were received from J. T. Bossé (Bossé et al., 2018).

Actinobacillus pleuropneumoniae strains were isolated on Tryptone Soya Agar (TSA, Biolab Ltd., Hungary) cross-inoculated with Staphylococcus aureus, and cultured on chocolate agar with added $50 \mu \mathrm{g} / \mathrm{ml}$ NAD (Biolab Ltd., Hungary), both containing $10 \%$ defibrinated sheep blood. Cultures were incubated at $37^{\circ} \mathrm{C}$ for $24 \mathrm{~h}$ in aerobic environment with the addition of $5 \%$ carbon dioxide. The $A$. pleuropneumoniae strains were identified using standard methods (Barrow and Feltham, 2003) and 16S rRNA PCR (Relman, 1993), and stored at $-80^{\circ} \mathrm{C}$ until further examination.

\section{Serological characterisation}

Hyperimmune sera were produced in rabbits against $A$. pleuropneumoniae reference strains of serovars $1-18$. Permission for the immunisation of rabbits was obtained from the Government Office of Pest County (PE/EA/3369-6/2016). For serotyping the indirect haemagglutination (IHA) test was performed as de- 
scribed by Biberstein (1978). All 18 type strains of A. pleuropneumoniae were tested with all antisera. Hyperimmune sera with the highest titre were used in the examination. All field strains of A. pleuropneumoniae were tested with all typing sera. Only those reactions were accepted where the titre of the haemagglutination reached that of the reference strain of the serotype and the cross reactions were also typical. Reactions under 1:160 titre were neglected.

\section{Results}

The 91 Hungarian isolates included in the present study were examined in the IHA test using hyperimmune sera raised against the 18 serovars of $A$. pleuropneumoniae. Out of these $91 \mathrm{~A}$. pleuropneumoniae strains 72 strains belonged to biotype I and 19 could be allocated to biotype II. The distribution of serotypes of A. pleuropneumoniae is shown in Table 1.

Table 1

Serotypes of Actinobacillus pleuropneumoniae in Hungary

\begin{tabular}{|c|c|c|c|c|c|c|}
\hline \multirow{2}{*}{ Serotype } & \multicolumn{2}{|c|}{ Before 2012} & \multicolumn{2}{|c|}{$2012-2016$} & \multicolumn{2}{|c|}{ Total } \\
\hline & strains & $\%$ & strains & $\%$ & strains & $\%$ \\
\hline 2 & 6 & 43 & 30 & 38.9 & 36 & 39.5 \\
\hline 5 & 0 & 0 & 1 & 1.3 & 1 & 1.1 \\
\hline 8 & 0 & 0 & 8 & 10.4 & 8 & 8.8 \\
\hline 9 & 1 & 7 & 4 & 5.2 & 5 & 5.5 \\
\hline 11 & 0 & 0 & 3 & 3.9 & 3 & 3.3 \\
\hline 12 & 0 & 0 & 3 & 3.9 & 3 & 3.3 \\
\hline 13 & 6 & 43 & 8 & 10.4 & 14 & 15.4 \\
\hline 14 & 1 & 7 & 0 & 0 & 1 & 1.1 \\
\hline 16 & 0 & 0 & 8 & 10.4 & 8 & 8.8 \\
\hline NT & 0 & 0 & 12 & 15.6 & 12 & 13.2 \\
\hline Total & 14 & 100 & 77 & 100 & 91 & 100 \\
\hline
\end{tabular}

NT: non-typable

\section{Discussion}

The results of these studies indicated that serotype 2 (39.5\%) and serotype $13(15.4 \%)$ strains of A. pleuropneumoniae were the most frequent in Hungary. Serotype 2 is dominant in most European countries (Dubreuil et al., 2000; Chiers et al., 2002; Maldonado et al., 2009; Stärk et al., 2007), but the high frequency of serotype 13 in Hungary is unique (Fodor et al., 1989; Gottschalk et al., 2003). All our serotype 13 strains belonged to biotype II. 
Untypable A. pleuropneumoniae strains could be isolated from clinical samples, and the frequency of the non-typable strains in Hungary is similar to the results of other authors (Eaves and Blackall, 1988; Gutiérrez et al., 1995; Klein et al., 2003; Morioka et al., 2016). Some non-typable strains could be allocated into clusters together with the serotypable ones indicating the possible loss of type-specific capsule polysaccharides (Kokotovic and Angen, 2007). Kokotovic and Angen (2007) described two clusters containing only non-typable A. pleuropneumoniae strains, and they supposed that these non-typable strains could represent additional serotypes. Further examinations can reveal whether our nontypable strains belong to one of these clusters.

As a result of previous serotyping using co-agglutination tests serotype 2 was dominant but serotypes $1,3,5,7,8,10,11$ and 12 also occurred in Hungary (Molnár, 1990) and serotype 13 was described at the same time (Fodor et al., 1989). Nowadays mainly serotypes $2,8,16$ and 13 are being isolated from clinical cases, but the isolation of non-typable strains has increased.

In conclusion, the IHA test is still one of the most sensitive classical serotyping methods. New molecular biological methods can be very helpful in serotyping $A$. pleuropneumoniae but they cannot completely replace the classical detection of antigens (Bossé et al., 2017, 2018). Serotyping is very important in the identification of $A$. pleuropneumoniae, since the haemagglutinating antigens of A. pleuropneumoniae are involved in the pathogenicity and in the protection against the disease caused by this agent. Regular monitoring for serotypes of $A$. pleuropneumoniae isolates is recommended because the frequency of the serotypes can change as a result of increased traffic of animals in the swine industry, thus necessitating potential adjustments in vaccine compositions.

\section{Acknowledgement}

This work was supported by the Hungarian Scientific Research Fund (OTKA 112826).

\section{References}

Angen, Ø., Ahrens, P. and Jessing, S. G. (2008): Development of multiplex PCR test for identification of Actinobacillus pleuropneumoniae serovars 1, 7 and 12. Vet. Microbiol. 132, 312-318.

Barrow, G. I. and Feltham, R. K. A. (eds) (2003): Cowan and Steel's Manual for the Identification of Medical Bacteria. Cambridge University Press, Cambridge. $331 \mathrm{pp}$.

Biberstein, E. L. (1978): Biotyping and serotyping of Pasteurella haemolytica. In: Bergan, T. and Norris, J. R. (eds) Methods in Microbiology 10. Academic Press, London. pp. 253-269.

Blackall, P. J., Klaasen, H. L. B. M., Bosch, H. V. D., Kuhnert, P. and Frey, J. (2002): Proposal of a new serovar of Actinobacillus pleuropneumoniae: serovar 15. Vet. Microbiol. 84, 47-52.

Bossé, J. T., Li, Y., Angen, Ø., Weinert, L. A., Chaudhuri, R. R., Holden, M. T., Williamson, S. M., Maskell, D. J., Tucker, A. W., Wren, B. W., Rycroft, A. N. and Langford, P. R. (2014): 
Multiplex PCR assay for unequivocal differentiation of Actinobacillus pleuropneumoniae serovars 1 to 3, 5 to 8, 10 and 12. J. Clin. Microbiol. 52, 2380-2385.

Bossé, J. T., Li, Y., Sárközi, R., Fodor, L., Lacouture, S., Gottschalk, M., Amoribieta, M. C., Angen, Ø., Nedbalcova, K., Holden, M. T. G., Maskell, D. J., Tucker, A. W., Wren, B. W., Rycroft, A. N. and Langford, P. R. (2018): Proposal of serovars 17 and 18 of Actinobacillus pleuropneumoniae based on serological and genotypic analysis. Vet. Microbiol. 217, 1-6.

Bossé, J. T., Li, Y., Sárközi, R., Gottschalk, M., Angen, Ø., Nedbalcova, K., Rycroft, A. N., Fodor, L. and Langford, P. R. (2017): A unique capsule locus in the newly designated Actinobacillus pleuropneumoniae serovar 16 and development of a diagnostic PCR assay. J. Clin. Microbiol. 55, 902-907.

Chiers, K., Donné, E., van Overbeke, I., Ducatelle, R. and Haesebrouck, F. (2002): Actinobacillus pleuropneumoniae infections in closed swine herds: infection patterns and serological profiles. Vet. Microbiol. 85, 343-352.

Chiers, K., de Waele, T., Pasmans, F., Ducatelle, R. and Haesebrouck, F. (2010): Virulence factors of Actinobacillus pleuropneumoniae involved in colonisation, persistence and induction of lesions in its porcine host. Vet. Res. 41, 65-81.

Dubreuil, J. D., Jacques, M., Mittal, K. R. and Gottschalk, M. (2000): Actinobacillus pleuropneumoniae surface polysaccharides: their role in diagnosis and immunogenicity. Anim. Health Res. Rev. 1, 73-93.

Eaves, L. E. and Blackall, P. J. (1988): Serological characterisation of Australian isolates of Actinobacillus pleuropneumoniae. Aust. Vet. J. 65, 379-381.

Fodor, L., Varga, J., Molnár, É. and Hajtós, I. (1989): Biochemical and serological properties of Actinobacillus pleuropneumoniae biotype 2 strains isolated from swine. Vet. Microbiol. 20, 173-180.

Frey, J., Beck, M., Bosch, J. V. D., Segers, R. P. A. M. and Nicolet, J. (1995): Development of an efficient PCR method for toxin typing of Actinobacillus pleuropneumoniae strains. Mol. Cell. Probes 9, 277-282.

Gottschalk, M. (2012): Actinobacillosis. In: Zimmerman, J. J., Karriker, L. A., Ramirez, A., Schwartz, K. J. and Stevenson, G. W. (eds) Diseases of Swine. Tenth edition. WileyBlackwell. Chichester, UK. pp. 653-669.

Gottschalk, M. (2015): The challenge of detecting herds sub-clinically infected with Actinobacillus pleuropneumoniae. Vet. J. 206, 30-38.

Gottschalk, M. and Lacouture, S. (2015): Canada: Distribution of Streptococcus suis (from 2012 to 2014) and Actinobacillus pleuropneumoniae (from 2011 to 2014) serotypes isolated from diseased pigs. Can. Vet. J. 56, 1093-1094.

Gottschalk, M., Broes, A., Mittal, K. R., Kobisch, M., Kuhnert, P., Lebrun, A. and Frey, J. (2003): Non-pathogenic Actinobacillus isolates antigenically and biochemically similar to Actinobacillus pleuropneumoniae: a novel species? Vet. Microbiol. 92, 87-101.

Grasteau, A., Tremblay, Y. D. N., Labrie, J. and Jacques, M. (2011): Novel genes associated with biofilm formation of Actinobacillus pleuropneumoniae. Vet. Microbiol. 153, 134-143.

Gutiérrez, C. B., Rodríguez Barbosa, J. I., Tascón, R. I., Costa, L., Riera, P. and Rodríguez Ferri, E. F. (1995): Serological characterisation and antimicrobial susceptibility of Actinobacillus pleuropneumoniae strains isolated from pigs in Spain. Vet. Rec. 137, 62-64.

Kamp, E. M., Vermeulen, T. M. M., Smits, M. A. and Jaagsma, J. (1994): Production of Apx toxins by field strains of Actinobacillus pleuropneumoniae and Actinobacillus suis. Infect. Immun. 62, 4063-4065.

Klein, C. S., Piffer, I. A., Ceroni da Silva, S., Schrank, A., Fávero, M. B. and Schrank, I. S. (2003): Detection of Actinobacillus pleuropneumoniae by PCR on field strains from healthy and diseased pigs. Curr. Microbiol. 46, 443-447.

Kokotovic, B. and Angen, Ø. (2007): Genetic diversity of Actinobacillus pneumoniae assessed by amplified fragment length polymorphism analysis. J. Clin. Microbiol. 45, 3921-3929. 
Maldonado, J., Valls, L., Martínez, E. and Riera, P. (2009): Isolation rates, serovars, and toxin genotypes of nicotinamide adenine dinucleotide-independent Actinobacillus pleuropneumoniae among pigs suffering from pleuropneumonia in Spain. J. Vet. Diagn. Invest. 21, 854-857.

Marois-Crehan, C., Lacouture, S., Jacques, M., Fittipaldi, N., Kobisch, M. and Gottschalk, M. (2014): Development of two real-time polymerase chain reaction assays to detect Actinobacillus pleuropneumoniae serovars 1-9-11 and serovar 2. J. Vet. Diag. Invest. 26, 146-149.

Marsteller, T. A. and Fenwick, B. (1999): Actinobacillus pleuropneumoniae disease and serology. Swine Health Prod. 7, 161-165.

Mittal, K. R., Higgins, R. and Lariviére, S. (1983a): Detection of type-specific antigens in the lungs of Haemophilus pleuropneumoniae-infected pigs by coagglutination test. J. Clin. Microbiol. 18, 1355-1357.

Mittal, K. R., Higgins, R. and Lariviére, S. (1983b): Determination of antigenic specificity and relationship among Haemophilus pleuropneumoniae serotypes by an indirect hemagglutination test. J. Clin. Microbiol. 17, 787-790.

Mittal, K. R., Higgins, R. and Lariviére, S. (1987): An evaluation of agglutination and coagglutination techniques for serotyping Haemophilus pleuropneumoniae isolates. Am. J. Vet. Res. 48, 219-226.

Mittal, K. R., Higgins, R. and Lariviére, S. (1988): Serologic studies of Actinobacillus (Haemophilus) pleuropneumoniae strains of serotype-3 and their antigenic relationships with other $A$. pleuropneumoniae serotypes in swine. J. Vet. Res. 49, 152-155.

Molnár, É. (1990): Survey of Actinobacillus (Haemophilus) pleuropneumoniae infection in swine by different methods. Acta Vet. Hung. 38, 231-238.

Morioka, A., Shimazaki, Y., Uchiyama, M. and Suzuki, S. (2016): Serotyping reanalysis of unserotypable Actinobacillus pleuropneumoniae isolates by agar gel diffusion test. J. Vet. Med. Sci. 78, 723-725.

Nielsen, R. (1986a): Serology of Haemophilus (Actinobacillus) pleuropneumoniae serotype 5 strains: establishment of subtypes A and B. Acta Vet. Scand. 27, 49-58.

Nielsen, R. (1986b): Serological characterization of Actinobacillus pleuropneumoniae strains and proposal of a new serotype: serotype 12. Acta Vet. Scand. 27, 453-455.

Nielsen, R., Andresen, L. O., Plambeck, T., Nielsen, J. P., Krarup, L. T. and Jorsal, S. E. (1997): Serological characterization of Actinobacillus pleuropneumoniae biotype 2 strains isolated from pigs in two Danish herds. Vet. Microbiol. 54, 35-46.

Ózsvári, L. and Búza, L. (2015): Comparative study on technology level, major production parameters and management of porcine respiratory disease complex (PRDC) in fattening pig herds [in Hungarian, with English abstract]. Magy. Allatorvosok 137, 79-92.

Perry, M. B., Angen, Ø., MacLean, L. L., Lacouture, S., Kokotovic, B. and Gottschalk, M. (2011): An atypical biotype I Actinobacillus pleuropneumoniae serotype 13 is present in North America. Vet. Microbiol. 24, 1-8.

Rayamajhi, N., Shin, S. J., Kang, S. G., Lee, D. Y., Ahn, J. M. and Yoo, H. S. (2005): Development and use of a multiplex polymerase chain reaction assay based on Apx toxin genes for genotyping of Actinobacillus pleuropneumoniae isolates. J. Vet. Diagn. Invest. 17, 359-362.

Relman, D. A. (1993): The identification of uncultured microbial pathogens. J. Infect. Dis. 168, 1-8.

Sárközi, R., Makrai, L. and Fodor, L. (2015): Identification of a proposed new serovar of Actinobacillus pleuropneumoniae: serovar 16. Acta Vet. Hung. 63, 444-450.

Stärk, K. D., Miserez, R., Siegmann, S., Ochs, H., Infanger, P. and Schmidt, J. (2007): A successful national control programme for enzootic respiratory diseases in pigs in Switzerland. Rev. Sci. Tech. 26, 595-606.

Zhou, L., Jones, S. C. P., Angen, Ø., Bossé, J. T., Nash, J. H. E., Frey, J., Zhou, R., Chen, H. C., Kroll, J. S., Rycroft, A. N. and Langford, P. R. (2008): Multiplex PCR that can distinguish between immunologically cross-reactive serovar 3, 6, and 8 Actinobacillus pleuropneumoniae strains. J. Clin. Microbiol. 46, 800-803. 